ADVERSE DRUG REACTION

\title{
Recurrent aseptic meningitis due to different non-steroidal anti-inflammatory drugs including rofecoxib
}

\author{
M L Ashwath, H P Katner
}

Postgrad Med J 2003;79:295-296

\begin{abstract}
Aseptic meningitis can be caused by viruses, drugs, and connective tissue disorders. The most common drugs causing it include antibiotics like trimethoprimsulfamethoxazole, non-steroidal anti-inflammatory drugs (NSAIDs), intravenous immunoglobulins, intrathecal agents, vaccines, and monoclonal antibodies. A patient who had aseptic meningitis from three different NSAIDs including rofecoxib is presented.
\end{abstract}

A 28 year old African-American woman with a past medical history of Sjogren's syndrome first presented in 1997 with complaints of headache, neck pain, fever, nausea, vomiting, photophobia, and blurred vision after taking naproxen for a week.

On examination she was confused, restless, and had nuchal rigidity. The remainder of the physical examination was normal. A spinal tap was done and the cerebrospinal fluid findings are shown in table 1 . Other studies on the cerebrospinal fluid including Gram stain, bacterial culture, venereal disease research laboratory test, cryptococcal antigen, pneumococcal antigen, acid fast bacilli smear and culture, India ink stain, and herpes polymerase chain reaction returned negative. Computed tomography and magnetic resonance imaging of the brain were normal. The patient was treated with ceftriaxone, vancomycin, and acyclovir empirically. She became more alert and comfortable two days after the naproxen was stopped, and was discharged on oral antibiotics.

The patient presented again in 1999 with similar complaints after taking ibuprofen for one week for sinusitis. Her physical examination was similar to the previous admission. The cerebrospinal fluid findings shown in table 1. All cerebrospinal fluid cultures, stains, and serologies were repeated and were negative. Computed tomography of her brain was read as normal. Her mental status and symptoms improved markedly two days after stopping the ibuprofen. She was initially treated with ceftriaxone and acyclovir, which were stopped after two days when she was thought to have aseptic meningitis secondary to non-steroidal anti-inflammatory drugs (NSAIDs). She was discharged with the advice not to use NSAIDs.

In December of 2001 she presented again with similar complaints and examination findings after taking rofecoxib for one week for joint pains. With the previous medical records initially unavailable, she was started again on ceftriaxone. Cerebrospinal fluid findings shown in table 1. The cultures were negative again and computed tomography of the brain was normal. The patient improved considerably two days after stopping rofecoxib and was discharged after further education.

\section{DISCUSSION}

Drug induced aseptic meningitis (DIAM) has been reported as an uncommon adverse reaction with numerous agents. ${ }^{1}$ It is a diagnosis of exclusion. A 1999 literature review reported fewer than 200 cases worldwide.

NSAID induced meningitis was first described in 1978 by Widener and Littman with ibuprofen in a patient with systemic lupus erythematosus. ${ }^{2}$ After what appeared to be the fourth temporally related episode of aseptic meningitis and ibuprofen ingestion, they electively admitted her to their clinical research centre and challenged her with a $400 \mathrm{mg}$ tablet dose of ibuprofen. She developed symptoms within a few hours which resolved within 36 hours. $^{2}$ Nine NSAIDs have been subsequently implicated in causing DIAM including naproxen, diclofenac, ketoprofen, sulindac, tolmentin, piroxicam, indomethacin, most commonly ibuprofen, ${ }^{3}$ and most recently rofecoxib. ${ }^{4}$

The clinical presentation is similar to that of meningitis due to infectious agents. Common systemic symptoms include fever, chills, nausea, vomiting, conjunctival irritation, facial oedema, stiff neck, stupor, delirium, and confusion. ${ }^{56}$ A high peripheral white blood cell count may be present. Spinal fluid findings include a pleocytosis, which can be several thousand

Abbreviations: COX, cyclo-oxygenase; DIAM, drug induced aseptic meningitis; NSAIDs, non-steroidal anti-inflammatory drugs

Table 1 Cerebrospinal fluid analysis in the three presentations

\begin{tabular}{|c|c|c|c|}
\hline Cerebrospinal fluid analysis & First admission 1997 & Second admission 1999 & Third admission 2001 \\
\hline White blood cells $/ \mathrm{mm}^{3}$ & 545 & 100 & 380 \\
\hline Neutrophils (\%) & 71 & 25 & 41 \\
\hline Lymphocytes (\%) & 24 & 64 & 54 \\
\hline Glucose $(2.2-3.9 \mathrm{mmol} / \mathrm{l})$ & $<1.1$ & 2.3 & 1.6 \\
\hline Protein $(0.15-0.45 \mathrm{~g} / \mathrm{l})$ & 1.58 & 0.86 & 0.87 \\
\hline Opening pressure $\left(\mathrm{mm}\right.$ of $\left.\mathrm{H}_{2} \mathrm{O}\right)$ & $>500$ & 277 & Not recorded \\
\hline Closing pressure $\left(\mathrm{mm}\right.$ of $\left.\mathrm{H}_{2} \mathrm{O}\right)$ & 257 & 154 & Not recorded \\
\hline Drug (dose) & Naproxen (250 mg twice a day) & Ibuprofen (400 mg three times a day) & Rofecoxib ( 25 mg every day) \\
\hline
\end{tabular}


cells. Usually there is a predominance of polymorphonuclear cells, though lymphocytic and eosinphilic findings are also reported. ${ }^{1}$ Most cases have a normal to low glucose, high protein, and high cerebrospinal fluid pressure. ${ }^{1356}$ Our patient demonstrated a spectrum of cerebrospinal fluid findings as recorded in table 1 . Whether this is due to the different NSAIDs or reflects a different stage in the disease process is yet to be determined.

The diagnosis is based on the close temporal relation between the use of the drug and the appearance of the symptomatolgy. It is important to exclude infectious and autoimmune causes. Lupus meningitis, sarcoidosis, and Behçet's syndrome may present similarly. The only way to confirm the diagnosis would be to rechallenge the patient with the same drug.

When DIAM is suspected, the drug should be promptly discontinued, and the patient should be given appropriate antimicrobials until infection is excluded.

The pathogenic mechanism of DIAM is still uncertain. Most authors believe it may be due to a hypersensitivity mechanism especially type III or type IV. ${ }^{17}$ This is supported by the temporal relationship between drug intake and the development of meningitis, prior exposure to the offending drug, and disappearance of symptoms after drug discontinuation. ${ }^{1}$ It has been proposed that the drug combines with a cerebrospinal fluid or meningeal protein that acts as a hapten leading to an inflammatory response in the meninges. ${ }^{1}$ Immune complex deposition, direct chemical irritation, immediate hypersensitivity, direct drug toxicity, and induction of antitissue antibodies have all been suggested. ${ }^{89}$

Though DIAM due to different NSAIDs in the same patient has been reported, many authors report an absence of cross reactivity of NSAIDs. This is based on their observation that patients who developed aseptic meningitis after exposure to one NSAID do not do so when subsequently treated with other NSAIDs. Our patient experienced this problem with three different classes of NSAIDs including the newest cyclooxygenase (COX) II inhibitor class. A recent case series has been reported of the first episode of meningitis with the COX II inhibitor rofecoxib. ${ }^{4}$ Our patient is, however, the first to demonstrate this cross reaction even with this new class of NSAIDs.
The other side effects in the central nervous system reported with NSAIDs include headache, hearing loss, tinnitus, psychosis, paranoid delusions, depersonalisation, hallucinations, cognitive dysfunction, and depression. ${ }^{5}{ }^{11}$

\section{CONCLUSION}

This case of aseptic meningitis due to rofecoxib illustrates that the potential for DIAM occurring with different classes of NSAIDs must also be considered for the new COX II inhibitor class. DIAM is a diagnosis of exclusion. Recognition and diagnosis is important as it is treatable by withdrawal of the drug and recurrence can be prevented. The outcome is generally good without long term sequelae.

\section{Authors' affiliations}

M L Ashwath, H P Katner, Department of Internal Medicine, Mercer University School of Medicine, Macon, Georgia

Correspondence to: Dr Ashwath; Mahi_Lakshmi@yahoo.com

Submitted 20 December 2002

Accepted 11 February 2003

\section{REFERENCES}

1 Moris G, Garcia-Monco JC. The challenge of drug-induced aseptic meningitis. Arch Intern Med 1999;159:1 185-92.

2 Widener $\mathbf{H}$ Littman B. Ibuprofen-induced meningitis in systemic lupus erythematosus. JAMA 1978;239:1062-4.

3 Jolles S, Sewell WA, Leighton C. Drug-induced aseptic meningitis: diagnosis and management. Drug Saf 2000;22:215-26.

4 Bonnel RA, Villalba ML, Karwoski CB, et al. Aseptic meningitis associated with rofecoxib. Arch Intern Med 2002;162:713-15.

5 Hoppmann RA, Peden GJ, Ober SK. Central nervous system side effects of nonsteroidal anti-inflammatory drugs. Arch Intern Med 1991:151:1309-13.

6 Peterson DI, Chong YB. Recurrent meningitis due to non steroidal anti-inflammatory drugs. Bull Clin Neurosci 1987;52:40-2.

7 Marinac JS. Drug and chemical induced aseptic meningitis. A review of literature. Ann Pharmacother 1992;26:813-22.

8 Von Reyn CF. Recurrent aseptic meningitis due to sulindac. Ann Intern Med 1988;99:343-4.

9 River Y, Averbuch-Heller L, Weinberger $M$, et al. Antibiotic induced meningitis. J Neurol Neurosurg Psychiatry 1994;57:705-8.

10 O'Brien WM, Babgy GF. Rare adverse reactions to nonsteroidal anti-inflammatory drugs. J Rheumatol 1985;12:785-90.

11 Jiang HK, Chang DM. Non-steroidal anti-inflammatory drugs with adverse psychiatric reactions: five case reports. Clin Rheumatol $1999: 18: 339-45$ 